\title{
Análise da alocação de mão-de-obra em linhas de multimodelos de produtos com demanda variável através do uso da simulação: um estudo de caso
}

\author{
Mariella Consoni Florenzano Souza, MSc. \\ E-mail:mariella@sc.usp.br \\ Mamoru Carlos Yamada, MSc. \\ E-mail:mcyamada@sc.usp.br \\ Arthur José Vieira Porto, Dr. \\ E-mail:ajvporto@sc.usp.br \\ Eduardo Vila Gonçalves Filho, Dr. \\ E-mail: evila@sc.usp.br \\ Escola de Engenharia de São Carlos - USP
}

\begin{abstract}
Resumo
A complexidade do planejamento e da programação de linhas de montagem de multimodelos de produtos, aliada ao seu comportamento dinâmico e à natureza estocástica dos tempos de montagem, sugere uma abordagem baseada na simulação para uma programação mais efetiva. Este trabalho utilizou a simulação para analisar a necessidade e alocação de mão-de-obra, considerando diferentes cenários, em função do número de montadores, do arranjo dos montadores na linha, da forma de trabalho dos montadores, da utilização das relações de paralelismo entre as atividades e da forma de entrada dos motores na linha. 0 estudo foi realizado em três linhas de sub-montagem de motores, de uma empresa do setor automobilístico, caracterizadas pela demanda diária variável, com data definida de entrega do produto acabado.
\end{abstract}

Palavras-chave

Linha de montagem, simulação, alocação de mão-de-obra, linha de multimodelos.

\section{Manpower allocation analysis in products multi-model lines with variable demand through the use of simulation: a case study}

\begin{abstract}
The complexity of planning and scheduling of multi-model assembly lines, joined to the dynamic behavior of these lines and the stochastic nature of the assembly times make a simulation based approach more effective to support the line scheduling. This work uses the simulation to analyze the manpower need and allocation, in different scenarios considering the number of operators, the operator's distribution on the workstations, the use of concurrency relationship of activities and the arrival sequence of engines in the line. This study was accomplished in three engines subassembly lines in a company of the automotive sector, characterized by a variable daily demand with defined delivery date for the finished products.
\end{abstract}

Key words

Assembly line, simulation, manpower allocation, multi-model line. 


\section{INTRODUĈ̣̃O}

No ambiente de mercado atual, os ciclos de vida dos produtos encontram-se cada vez menores e a demanda por uma maior variedade de produtos está cada vez mais freqüente. Neste contexto, as empresas estão passando a produzir vários modelos de um produto em uma mesma linha de montagem, em lotes reduzidos e visando satisfazer o mercado em menores lead times. Assim, surgem as linhas de montagem de multimodelos de produtos, caracterizadas por uma maior flexibilidade quanto à seqüência das atividades de montagem e quanto à possibilidade de fabricar os modelos demandados no dia pelo mercado e na quantidade adequada.

Por outro lado, o planejamento e a programação da produção para as linhas de multimodelos normalmente são mais complexos, pois o mix a ser produzido e as respectivas quantidades mudam diariamente, afetando os tempos de montagem em cada estação. Em muitas ocasiões, a linha pode tornar-se desbalanceada, e atingir uma boa taxa de utilização do montador pode ser uma tarefa difícil.

A flexibilidade destas linhas, adicionada ao seu comportamento dinâmico, às propriedades dos dados estocásticos dos tempos de execução das atividades e à possibilidade da realização de atividades em paralelo, faz com que as empresas busquem ferramentas alternativas de suporte ao planejamento e programação da produção como, por exemplo, uma abordagem baseada na simulação, visando analisar a resposta de diversos fatores tais como a seqüência de entrada de modelos na linha e a quantidade de montadores, no atendimento à demanda.

A simulação de sistemas discretos permite que as alterações no mix de produção possam ser analisadas e as atualizações necessárias possam ser identificadas e realizadas com maior efetividade, tornando o sistema mais ágil para responder às variações de necessidades do mercado.

O objetivo do trabalho em questão é utilizar a simulação para analisar a necessidade e alocação de mão-deobra em três linhas de submontagem de multimodelos de motores de uma empresa do setor automobilístico, caracterizadas pela demanda diária variável, com data definida de entrega do produto acabado. O estudo considerou diferentes possibilidades de simulação da linha para o atendimento da previsão de demanda das linhas principais de montagem, em função do número de montadores, do arranjo dos montadores na linha, da forma de trabalho dos montadores, da utilização das relações de paralelismo entre as atividades e da forma de entrada dos motores na linha.

\section{CARACTERIZAC̣̃̃O DE LINHAS DE MONTAGEM DE MULTIMODELOS DE PRODUTOS}

Em um sistema de linha de montagem, a matériaprima entra e progressivamente se move através de uma série de estações de trabalho enquanto está sendo transformada no produto desejado. O conteúdo total de trabalho do processo de montagem é dividido entre as estações de trabalho, sem violar as relações de precedência de montagem. De acordo com Askin; Standridge (1993), esse tipo de sistema de manufatura tende a ser um dos mais eficientes, porém requer processos confiáveis, com mínima variabilidade nos tempos de processamento nas estações de trabalho e entre elas, e nos tipos de produtos que estão sendo produzidos. Além disso, um ambiente desejado para a linha de montagem é aquele com alto volume de produção de um único produto.

Na prática, muitos produtos não possuem demanda suficiente para justificar uma linha de montagem, principalmente em um ambiente onde a demanda se caracteriza pela grande diversidade de modelos do produto e por ciclos de vida reduzidos. A montagem de diferentes modelos em pequenos lotes, e curtos lead times, além da utilização de produtos modulares têm levado à utilização de linhas flexíveis, como as de multimodelos de produto, nas quais diferentes modelos do produto são produzidos na mesma linha, separadamente em lotes.

As linhas de multimodelos apresentam uma maior flexibilidade principalmente com relação à sequiência das atividades de montagem, que pode ser diferente de um modelo para o outro, pois mesmo que algumas atividades sejam comuns para os modelos, nem sempre elas são realizadas na mesma seqüência (COSTA; FERREIRA, 1999). Além disso, a flexibilidade pode também ser mencionada quanto à possibilidade de montar os modelos demandados pelo mercado no dia e na quantidade certa. Em outras palavras, estas linhas possibilitam satisfazer a demanda diária e ajustar a produção rapidamente sem a necessidade de grandes estoques de produtos finais e, conseqüentemente, reduzindo-se os custos de inventário (SIANESI, 1998).

Essa flexibilidade, porém, pode resultar em uma programação da produção mais complexa, sendo que o mix de modelos a ser produzido e as respectivas quantidades mudam diariamente. Os tempos de montagem em cada estação, para cada modelo, podem ser diferentes, sendo que a quantidade de trabalho requerido de um operador não é necessariamente a mesma para todas as estações. Além disso, quando o volume de produção não é tão elevado, não há a necessidade de se alocar um montador para cada estação de trabalho, ou seja, ele pode realizar 
atividades em mais de uma estação de trabalho. Nesses casos, a empresa pode se deparar com uma linha desbalanceada e pode encontrar dificuldades para atingir um bom nível de utilização dos montadores.

Ghosh; Gagnon (1989) apresentam uma revisão bibliográfica e uma análise sobre o projeto, balanceamento e programação de linhas de montagem, para linhas simples, de multimodelos e de modelos mistos. Os autores levantam fatores qualitativos e quantitativos que influenciam tais linhas. Ho et al. (1993) propõem dois métodos de análise para o projeto de arranjo físico de linhas de multiprodutos. He \& Kusiak (1998) propõem uma abordagem para o projeto de linhas de montagem para produtos modulares que divide a linha em duas linhas de submontagem: uma para operações básicas e outra para operações variantes de montagem, considerando os problemas de balanceamento de linha e programação. Fokkert; Kok (1997) comparam e analisam diferentes heurísticas para balanceamento de linhas de multimodelos e de modelos mistos, no desempenho das linhas. Khan; Day (2002) propõem uma metodologia de projeto baseada no conhecimento tanto para linhas automatizadas quanto para linhas manuais, que pode ser aplicada igualmente para linhas que montam um único produto, linhas de multimodelos ou linhas de modelos mistos. Além disso, a metodologia proposta pode ser aplicada tanto para tempos determinísticos quanto para tempos estocásticos.

Uma escolha importante na seleção de uma linha de montagem é se a linha será síncrona ou assíncrona (ASKIN; STANDRIDGE, 1993). Em uma linha síncrona, cada estação de trabalho possui exatamente a mesma quantidade de tempo para trabalhar cada unidade do produto. No final de cada ciclo de tempo, o sistema de movimentação automaticamente leva cada unidade para a próxima estação. As linhas síncronas podem evitar a necessidade de remover e substituir unidades de produto do mecanismo de transporte em cada estação, ajudar a motivar os trabalhadores a manter o ritmo e o sincronismo e contribuir para o balanceamento da produção. No entanto, a aleatoriedade do desempenho ocasionalmente fará com que alguns itens não sejam completados. Assim, tempo extra deverá ser permitido no tempo de ciclo fixo para compensar a variabilidade do tempo de tarefa. Já nas linhas assíncronas, a estação remove uma nova unidade do sistema de movimentação assim que ela tenha completado a unidade anterior, desempenha as tarefas requeridas, e então envia a unidade para a estação seguin- te. As linhas assíncronas possuem a vantagem, em geral, de permitir alguma variabilidade nos tempos das estações enquanto minimiza o tempo de fluxo. As linhas de multimodelos de produto geralmente são assíncronas, para permitir que estações compensem o tempo perdido em um item de trabalho intensivo quando entrar um item de menor conteúdo de trabalho na linha. a necessidade e alocação de mão-de-obra

\section{em três linhas de submontagem de} multimodelos de motores de uma empresa do setor automobilístico.

Uma linha pode ser projetada considerando-se tempos determinísticos ou estocásticos. Geralmente, os tempos de operação em linhas manuais não são constantes, pois variam a cada vez que uma operação é realizada. Estas variações podem introduzir irregularidades no fluxo ao longo da linha, o que, por sua vez, podem causar dois efeitos: criação de filas temporárias e perda de tempo disponível de trabalho. Pode até ser necessário introduzir mais recursos na operação para compensar a perda de eficiência resultante da variação dos tempos de trabalho.

Neste contexto, a natureza complexa das linhas de multimodelos, dificulta o seu controle, a sua programação diária e a alocação da mão-de-obra necessária para executar o trabalho de montagem. Essa dificuldade, por sua vez, leva as empresas a buscarem técnicas de análise de diferentes alternativas de funcionamento da linha, que possibilitem prever seu comportamento sob diversas condições, visando obter melhores soluções. Uma abordagem baseada na simulação apresenta-se como uma poderosa técnica de apoio ao desenvolvimento dessas atividades, pois permite analisar e comparar as diferentes formas de programação da linha e alocação da mão-deobra, mostrando a influência dos diversos fatores e incorporando o caráter estocástico dos processos envolvidos.

\section{APLICAC̣̃̃ES DA SIMULAC̣ÃO EM LINHAS DE MONTAGEM}

A simulação da manufatura pode ser usada como uma técnica de análise para prever o efeito de modificações em um sistema existente ou como uma técnica de apoio ao projeto, para avaliar o desempenho de um novo siste- 
ma sob uma ampla variedade de circunstâncias. O comportamento de um sistema, durante sua evolução ao longo do tempo, pode ser estudado por meio do desenvolvimento de um modelo, o qual toma a forma de um conjunto de considerações relacionadas à operação do sistema. Uma vez desenvolvido e validado, o modelo pode ser usado para investigar uma grande variedade de perguntas do tipo "e se" sobre o sistema real.

a simulação foi utilizada para testar diferentes seqüências e regras de programação, implementadas no programa de controle da linha. Vaidyanathan et al. (1998) descrevem a aplicação da simulação como uma ferramenta de programação da produção diária numa indústria de processamento de café. Uma abordagem de análise de mix de produto com simulação foi apresentada por Al-Aomar (2000), na qual utiliza a simulação para validar o ponto ótimo de um modelo prévio em programação linear, ou apontar melhorias nas configurações de modo a auxiliar na obtenção do ponto ótimo.

Assim, um modelo de simulação pode ser utilizado para: assegurar que um sistema de manufatura validado encontre seus requisitos ao longo de prolongados períodos de tempo; dar suporte às mudanças para a realização de melhorias contínuas; auxiliar na solução do planejamento e programação do sistema; avaliar a utilização dos recursos humanos necessários; auxiliar o treina-

Áreas de notáveis aplicações da simulação no campo da manufatura incluem a simulação do arranjo físico de fábrica, do fluxo de processo, dos sistemas de manuseio de material, do planejamento de capacidade, da utilização de mão-de-obra, do investimento em novos equipamentos, e da programação de produção, entre outras. Outras aplicações estão relacionadas a logística, configuração de ferramentas, programação $\mathrm{CN}$ e treinamento.

Tradicionalmente, a simulação tem sido bastante aplicada ao planejamento, projeto e análise de longo prazo de linhas de montagem. Durante o projeto, a simulação é, muitas vezes, usada nas primeiras etapas para ajudar a compreender a forma de funcionamento do sistema. Pode também ser usada para validar e verificar a solução final, assegurando que o projeto da linha, quando implementado, irá alcançar os objetivos identificados. Fuh et al. (1996) realizam um estudo de simulação visando analisar um novo sistema automatizado de montagem de produtos computacionais de alta precisão e avaliar o desempenho do sistema entre diferentes alternativas de projeto. Artto et al. (2001) sugerem a utilização da simulação de eventos discretos como uma ferramenta de gerenciamento de projeto, que permite o gerenciamento contínuo da funcionalidade do projeto ainda durante sua implementação.

Outra aplicação importante da simulação é como suporte ao controle e operação da linha. Neste aspecto, Costa; Ferreira (1999) relatam o estudo, simulação e avaliação do desempenho de uma linha flexível, no qual mento e educação do pessoal envolvido na operação do novo sistema.

No caso de uma linha de montagem de multimodelos de produtos, a complexidade proveniente da maior flexibilidade e da natureza estocástica da duração das operações possibilitam uma abordagem baseada na simulação para alcançar uma solução para o melhor balanceamento do trabalho da mão-de-obra, principalmente se a demanda por modelo de produto é variável e se a data de entrega é definida. Bengu (1994) estuda uma linha em fluxo flexível, concluindo que técnicas normalmente usadas para a programação diária da produção ignoram algumas dinâmicas inerentes e propõem uma programação baseada na simulação.

\section{ESTUDO DE CASO}

A seguir, será apresentado um estudo de caso realizado em uma empresa do setor automobilístico. No estudo foi utilizada uma ferramenta de simulação com o objetivo de analisar a alocação de mão-de-obra em três linhas de montagem de multimodelos de produtos com demanda variável e data de entrega definida. Para resguardar o sigilo das informações da empresa, os nomes de motores, atividades, etc., foram substituídos por letras ou números.

\section{Apresentação do problema}

Uma reestruturação no setor de sub-montagem da 
empresa em questão gerou uma mudança do layout posicional para um layout por produto ou em linha. Os módulos (motores, tanques, cabines, etc.), que eram montados ao lado das linhas principais de montagem, passaram, dessa forma, a ser montados em linhas modulares de submontagem. Assim, cada módulo passa a ter a sua própria linha de submontagem. Tal modificação visou proporcionar, entre outros benefícios, linhas de montagem mais curtas, um maior sinergismo de competências para os módulos, a redução do número de ferramentas, e um menor tempo nas linhas principais de montagem.

Uma das linhas modulares geradas foi a de submontagem de motores, na qual diversos subsistemas e peças são anexados ao motor, formando um módulo mais completo. Como a demanda individual de cada modelo de motor e o conteúdo de trabalho envolvido na sua montagem não justificavam uma linha de submontagem dedicada a cada modelo, foram projetadas três linhas (1, 2 e 3 ), cada uma responsável pela montagem de um conjunto de diferentes modelos de motores, de acordo com a semelhança das seqüências e tempos das atividades de montagem. Tem-se, portanto, linhas de multimodelos de motores. Mesmo assim, uma das linhas de submontagem de motores (linha 3) não justificaria a alteração de um layout posicional para o layout em linha, considerandose o volume atual dos modelos montados nesta linha. Porém, uma análise da previsão de demanda futura indicou um provável aumento da demanda, além da inclusão de um novo modelo de motor a ser montado na linha.

A linha 1 é composta por seis estações de trabalho e é responsável pela submontagem de quatro modelos de motores (A, B, C e D). A linha 2, a qual monta também quatro modelos de motores (E, F, G e H), é formada por cinco estações de trabalho. E, por fim, a linha 3, que é composta por quatro estações de trabalho, é responsável pela submontagem de três modelos de motores (I, J e K).

As linhas de submontagem foram projetadas para funcionarem de forma assíncrona, permitindo alguma flexibilidade em relação aos tempos de montagem em cada estação de trabalho e para cada tipo de motor. A movimentação dos motores de uma estação para outra é feita pelos próprios montadores, que empurram o motor (apoiado em um carrinho) para a próxima estação, assim que completam suas atividades.

Um dos problemas encontrados, principalmente devi- do ao baixo volume demandado de cada modelo de motor e à variação mensal e diária da demanda, foi a alocação de mão-de-obra nas estações de trabalho, sendo que muitas vezes não era necessária a utilização de um operário por estação de trabalho.

Além disso, foram identificados dois pontos críticos nesta nova concepção de montagem: o primeiro é que as linhas modulares devem atender a demanda das linhas principais de montagem e devem estar em sincronismo com as mesmas, a fim de evitar estoques excessivos entre elas; o segundo ponto crítico consiste na grande diversidade de modelos de motores, que dificulta a programação da fábrica. Dessa forma, foi identificada a necessidade de um cálculo mais preciso da quantidade mensal de mãode-obra.

As linhas de submontagem possuem algumas características que devem ser consideradas. Uma delas é que o mix de modelos muda diariamente conforme a demanda diária das linhas principais de montagem, dificultando o balanceamento e a programação da linha. Uma outra característica é a presença de diferentes estruturas do produto para cada modelo, tanto em termos de seqüência de atividades, quanto em termos de duração da montagem. Mesmo que algumas das operações sejam comuns a mais de um modelo de produto, não são necessariamente realizadas seguindo a mesma seqüência.

\section{ma abordagem baseada na simulação permite analisar e comparar as diferentes formas de programação da linha e alocação da mão-de-obra, mostrando a influência dos diversos fatores e incorporando o caráter estocástico dos processos envolvidos.}

Portanto, o objetivo do estudo de simulação foi analisar o comportamento dinâmico das linhas de submontagem de motores projetadas, visando identificar a necessidade e o balanceamento de mão-de-obra por estação de trabalho; e sincronizar a linha de submontagem com as linhas principais de montagem, procurando minimizar o estoque de motores prontos entre estas etapas.

Para a realização do estudo, as seguintes condições de contorno foram fornecidas pela empresa: o estoque pretendido de motores prontos aguardando a demanda da linha principal é de um dia; a entrada de motores na linha de submontagem não deve ultrapassar três dias de ante- 
cedência em relação à entrada do motor na linha principal de montagem; e deve-se evitar, sempre que possível, a utilização dos segundos e terceiros turnos, horas extras e banco de horas. Considerando estas restrições fornecidas pela empresa e o atendimento da demanda diária de cada modelo de produto, o trabalho visou analisar a necessidade e alocação da mão-de-obra, racionalizando a utilização dos montadores alocados para a execução das atividades de submontagem.

O procedimento de desenvolvimento do estudo de simulação das três linhas de submontagem de motores abrangeu os seguintes estágios: análise e planejamento, modelagem, programação, execução dos experimentos e apresentação e análise dos resultados.

\section{Análise e Planejamento}

$\mathrm{Na}$ fase de Análise e Planejamento, o estudo de simulação para o mix de produção foi planejado para as três linhas de submontagem, tendo como objetivo o dimensionamento da mão-de-obra, quanto à quantidade e à forma de alocação, necessária para o atendimento da previsão de demanda das linhas principais de montagem.

Considerou-se que os modelos deveriam ser "neutros" em relação a quaisquer softwares de simulação, ou seja, os modelos (teóricos) deveriam permitir a programação em qualquer linguagem de simulação ou sistema que viesse a ser futuramente definido ou alterado.

No estudo, considerou-se também a possibilidade da utilização de cobertura de mão-de-obra, ou seja, a possibilidade de se trabalhar com um montador realizando atividades que podem ser executadas em paralelo com outras na mesma estação, não interferindo na sua realização.

\section{Modelagem}

$\mathrm{Na}$ fase de Modelagem, o sistema foi definido e detalhado tanto em relação aos experimentos realizados quanto em relação às características de alcance (amplitude do modelo ou quanto do sistema o modelo deve conter), precisão (exatidão dos dados que estão sendo utilizados para a construção do modelo), restrição, relação de concorrência (pela mão-de-obra e recursos), paralelismo (pelo uso da mão-de-obra ou de recursos) e seqüencialidade das atividades (seqüência mínima obrigatória para atendimento aos requisitos do produto). Todas as atividades foram modeladas em relação às suas propriedades e definidas quanto à natureza (contínua, estocástica, etc.), e os respectivos valores foram modelados e coletados.

A fase de modelagem é composta pelas seguintes tarefas:

- Modelagem das atividades: realizou-se a descrição das atividades e seus respectivos tempos de montagem em cada estação, por tipo de motor.

- Modelagem das estações de trabalho: foram definidos os relacionamentos entre as atividades, em relação às seqüencialidades e possibilidades de paralelismos. A modelagem foi realizada utilizando-se diagramas de precedência. Como exemplo, a Fig. 1 apresenta o diagrama desenvolvido para o motor I da linha 3. Este motor possui 29 atividades de montagem, divididas entre as quatro estações de trabalho. Na estação 1 , as atividades 1 e 2 podem ser realizadas em paralelo, mas ambas devem ser finalizadas para que a atividade 3 possa ser iniciada. Após o término da atividade 3 , as atividades $4,5,6$ e 7 podem ser iniciadas paralelamente.

- Modelagem das linhas: foi realizada a modelagem do arranjo físico das linhas, com relação às estações de trabalho, e ao conjunto de atividades de cada estação, obedecendo-se às regras de precedência. Assim, foram definidos os conjuntos de atividades que serão realizados em cada estação de trabalho, considerando-se o balanceamento nos tempos totais de montagem para cada estação.

- Modelagem dos dados: procurou-se agregar aos modelos uma característica de flexibilidade em relação às altera-

Figura 1: Diagrama de precedência do motor I da linha 3.

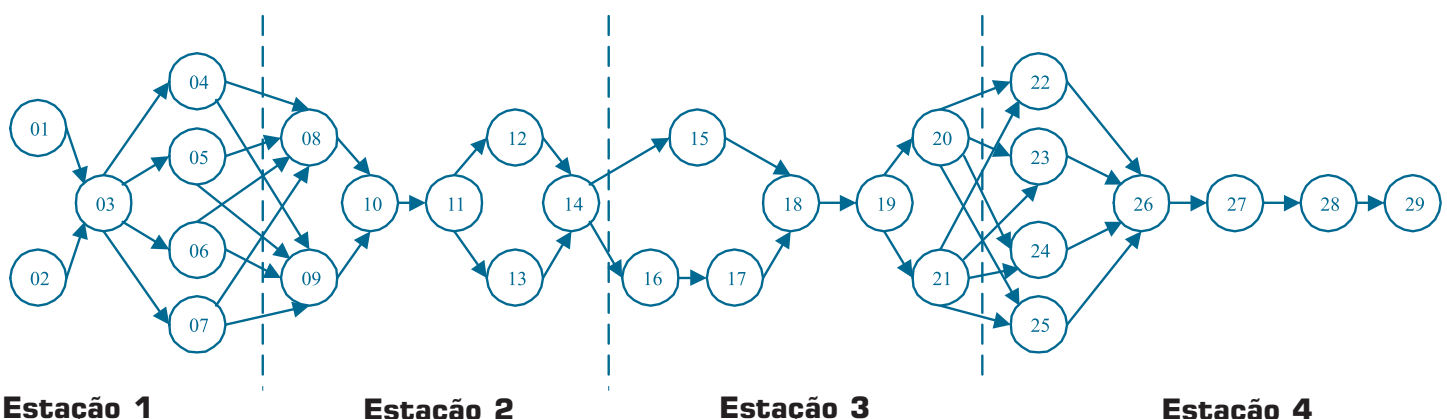


ções nos dados de projeto fornecidos pela empresa, que seriam os dados de entrada do modelo: tempos e sequiência de atividades, e programação de demanda mensal das linhas principais de montagem. Para tanto, geraram-se arquivos externos ao modelo, nos quais estas informações são armazenadas, podendo sofrer modificações e atualizações. Esses arquivos são lidos pelo software de simulação utilizado. Juntamente com os dados de entrada, foram levantadas na empresa as principais condições de contorno do projeto:

- Os tempos de montagem para cada atividade apresentam uma incerteza de 10\%. A empresa possuía tempos-padrões de montagem, que foram auditados por uma empresa externa. Como não possuía levantamentos estatísticos dos tempos de montagem, ela adotou como dado de projeto o valor de $10 \%$ dos tempos-padrões como a variação máxima permitida nos tempos de montagem, para mais ou para menos. Assim, em função da indisponibilidade de dados para a modelagem desta variação, optou-se por modelar os tempos de montagem por meio de uma distribuição triangular, na qual:

- a moda é o valor dos tempos-padrões auditados;

- o mínimo valor é $90 \%$ do valor dos tempos-padrões auditados;

- o máximo valor é $110 \%$ do valor dos tempospadrões auditados.

- Rendimento global de $60 \%$ para dois montadores trabalhando juntos nas mesmas atividades. Segundo a empresa estudada, a experiência mostrava que alocar dois montadores para realizar a mesma atividade de montagem não implica numa redução de $50 \%$ nos tempos padrões de montagem. A empresa observou que, na prática, ao trabalharem juntos numa mesma atividade, dois montadores conseguem uma redução para $60 \%$ dos tempos padrões de montagem. Ou seja, se um montador leva, em média, 10 minutos para executar uma determinada atividade, dois montadores trabalhando juntos levarão 6 minutos para concluir essa mesma atividade.

- Turno diário de 8,8 horas.

\section{montagem de motores abrangeu os seguintes estágios: análise e planejamento, modelagem, programação, execução dos experimentos e apresentação e análise dos resultados.}

- Detalhamento dos experimentos: para cada linha, definiu-se a construção de modelos para o estudo de mix de montagem, considerando-se os dados de entrada fornecidos pela empresa. Os experimentos a serem executados deveriam verificar e comparar diversas configurações de mão-de-obra (quantidade e forma de trabalho), em relação ao atendimento da previsão de demanda. Assim, numa análise inicial, considerou-se que, para cada estação, poderiam trabalhar até três montadores ao mesmo tempo, sendo que dois na mesma atividade, e um em uma atividade paralela (sistema de cobertura). Para as linhas de baixa demanda (linhas 2 e 3), onde não são necessários montadores em todas as estações, o arranjo de trabalho foi considerado, isto é, os montadores poderiam trabalhar de várias maneiras: em todas as estações, em estações específicas, poderia haver estações com um montador e outras com dois, entre outras.

Figura 2: Diagrama de alocação de montadores para o experimento 1.

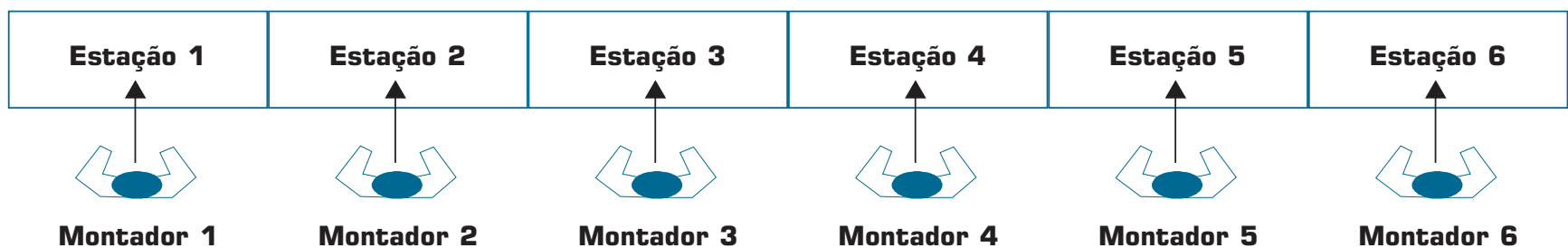




\section{Programação}

Após a fase de modelagem, foi realizada a Programação das linhas. As atividades realizadas nesta fase foram:

- Estruturação do modelo neutro na linguagem de simulação: a programação da modelagem das linhas foi executada utilizando-se o software de simulação AutoMod, da Autosimulations, na versão 10.0. O ambiente de simulação deste software representa as dimensões da linha de submontagem na mesma escala do arranjo físico fornecido pela empresa.

- Programação da linha: foram elaboradas programações específicas para cada tipo de motor, onde os tempos de montagem e as relações de paralelismo entre as atividades foram lidos de arquivos de entrada de dados. A previsão de demanda também foi programada para ser lida de outro arquivo de dados que armazena o tipo dos motores, o dia e a sequiência de entrada dos motores na linha de submontagem.

A programação das linhas de submontagem para o estudo de mix de produção foi desenvolvida considerandose a programação de demanda mensal e diária, de acordo com a previsão das linhas principais de montagem. Os resultados analisados, alterando-se a configuração da mão-de-obra, foram: a identificação de gargalos, a taxa de ocupação da mão-de-obra e o atendimento da demanda.

\section{Execução dos Experimentos}

$\mathrm{Na}$ fase de Execução dos Experimentos, várias possibilidades de simulação da linha para o atendimento da previsão de demanda das linhas principais de montagem foram identificadas, em função do número de montadores, do arranjo dos montadores na linha, da forma de trabalho dos montadores, da utilização das relações de paralelismo entre as atividades e da forma de entrada dos motores na linha. Tais possibilidades foram simuladas e analisadas de acordo com as condições de contorno fornecidas pela empresa, já mencionadas no item Modelagem.

Dos diversos experimentos realizados, foram selecionados, para cada linha de montagem, aqueles que melhor atenderam à previsão de demanda.

$\mathrm{Na}$ linha 1, a demanda programada de motores tem volume suficiente para que a linha funcione em regime contínuo. Neste caso, as possibilidades de simulação da linha consideraram, no mínimo, um montador por estação de trabalho. Com este ponto de partida, foram analisadas e simuladas várias alternativas, envolvendo as possibilidades de cobertura e de trabalho conjunto de dois montadores numa mesma estação de trabalho. Dentre todas essas possibilidades, o experimento que apresentou melhores resultados foi:

- Experimento 1: um montador por estação de trabalho, totalizando, dessa forma, seis montadores (Figura 2).

Para as linhas 2 e 3, o volume da demanda programada mostrou-se insuficiente para que estas linhas alcancem o estado de regime contínuo de produção. Desta forma, as possibilidades de simulação das linhas consideraram um número de montadores sempre menor que o número de estações. Para estas linhas, também foram analisadas e simuladas várias alternativas, que envolveram, além das possibilidades de cobertura e do trabalho conjunto de dois montadores numa mesma estação de trabalho, o número de montadores na linha.

Para a linha 2, dois experimentos apresentaram melhores resultados. Ambos utilizam dois montadores, porém o arranjo de trabalho é diferente:

- Experimento 2: dois montadores, sendo que cada montador trabalha em um motor, sendo responsável pela montagem total do motor (Figura 3).

- Experimento 3: dois montadores trabalhando juntos nas mesmas atividades, considerando um rendimento de $60 \%$ (Figura 4).

Para a linha 3, o experimento com melhores resultados foi:

- Experimento 4: três montadores, sendo que dois realizam simultaneamente as mesmas atividades nas estações 1 e 2 (gargalos), e um trabalha nas estações 3 e 4 (Figura 5).

\section{Apresentação e análise dos resultados}

Para cada linha de submontagem de motores, os resultados obtidos na execução dos experimentos são apresen-

Figura 3: Diagrama de alocação de montadores para o experimento 2.

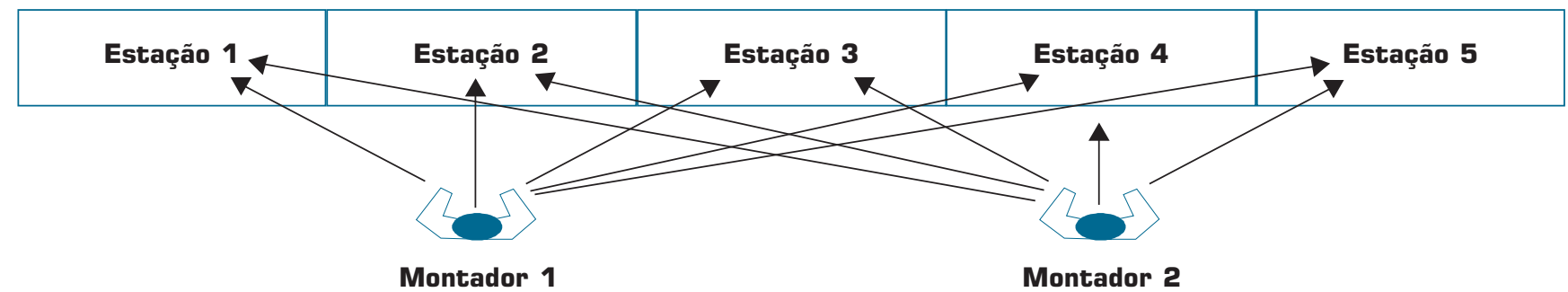


tados e analisados a seguir. Em todos os experimentos, os resultados foram obtidos sem a utilização do segundo e terceiro turnos, horas extras e banco de horas.

\section{Linha 1}

Para cada execução dos experimentos descritos anteriormente, apresentou-se uma sugestão de programação diária da linha para atendimento da demanda, junto com as taxas de ocupação diária dos montadores. A sugestão de programação gerada pela execução do experimento 1 , para a linha 1 , está representada na Tabela 1.
Esta tabela apresenta, na parte superior, os dias úteis da programação de montagem da linha principal. A seguir, para cada coluna de dia útil, tem-se quatro subcolunas nas quais pode-se acompanhar a entrada e a saída de motores na linha de submontagem. As subcolunas "QE" e "QS"mostram, respectivamente, a quantidade de um determinado motor que está entrando e saindo da linha em um determinado dia. As subcolunas "E" e "S" identificam, respectivamente, o tipo de motor que está entrando e saindo da linha em um determinado dia, por meio de um código que contém uma letra e um número entre

Figura 4: Diagrama de alocação de montadores para o experimento 3.

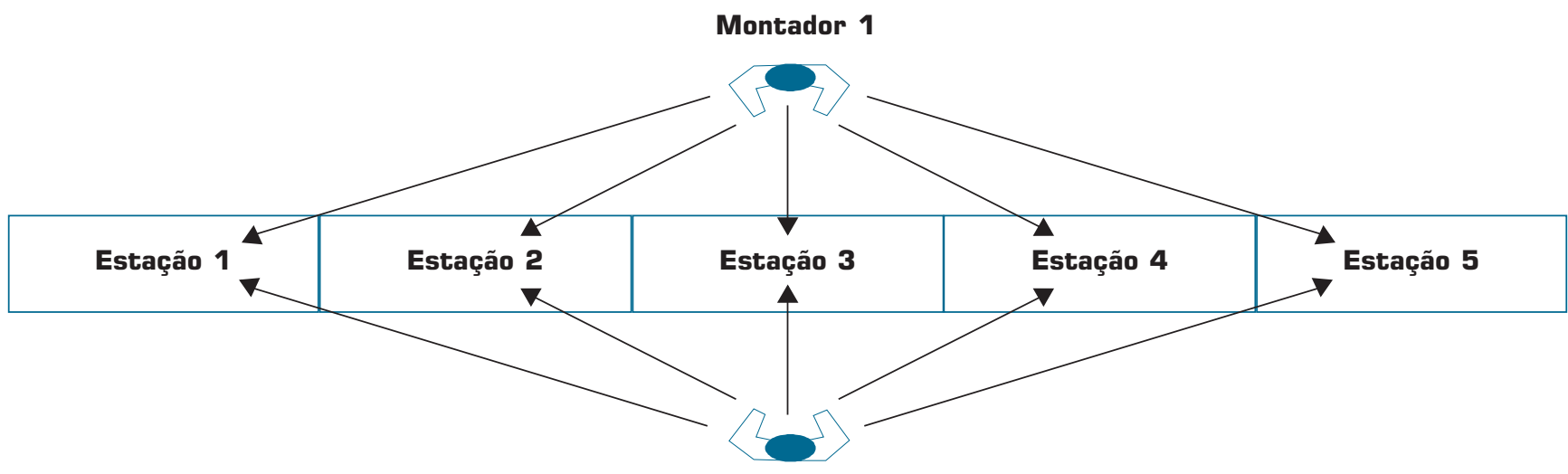

Montador 2

Figura 5: Diagrama de alocação de montadores para o experimento 4.

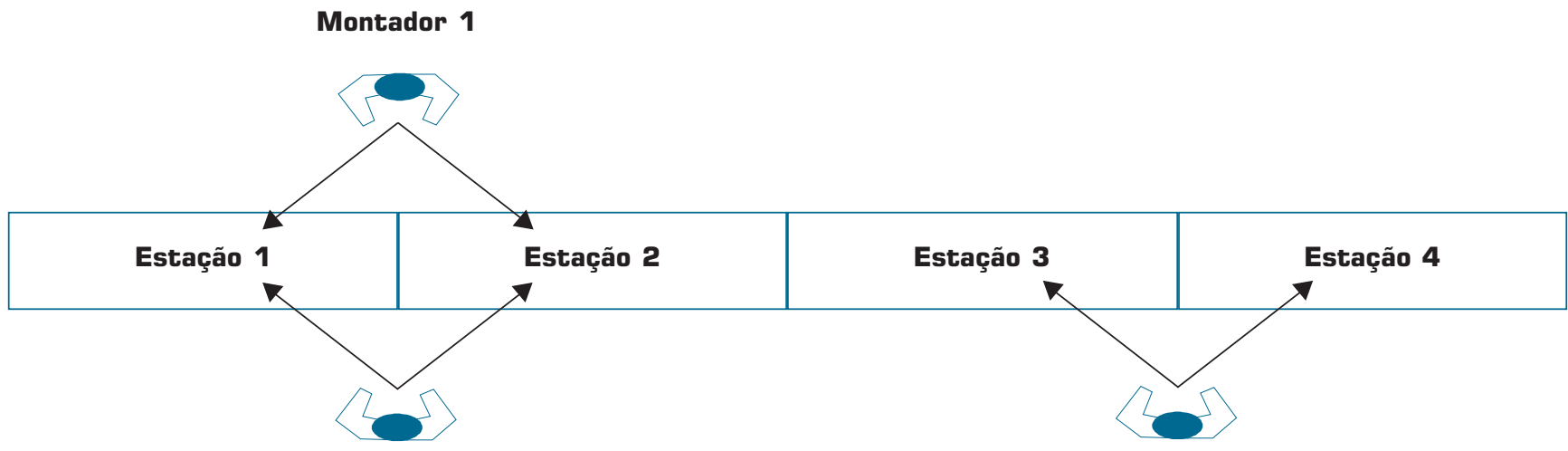

Montador 2

Montador 3 
parênteses (por exemplo: A(1)). A letra representa o tipo do motor e o número representa o dia útil em que o motor deve estar pronto para a montagem final na linha principal. Desta forma, o motor pode ser acompanhado desde o seu dia de entrada e de saída da linha de submontagem até o seu dia de entrada na linha principal de montagem.
Finalmente, a tabela apresenta, na parte inferior, a taxa de ocupação diária de cada montador. Exemplificando: com dois dias de antecedência do primeiro dia útil da programação de montagem na linha principal, entram na linha 1 de submontagem, cinco motores do tipo A, dois do tipo $\mathrm{B}$, um do tipo $\mathrm{C}$, dois do tipo E e novamente três

Tabela 1: Sugestão de programação mensal para a linha 1.

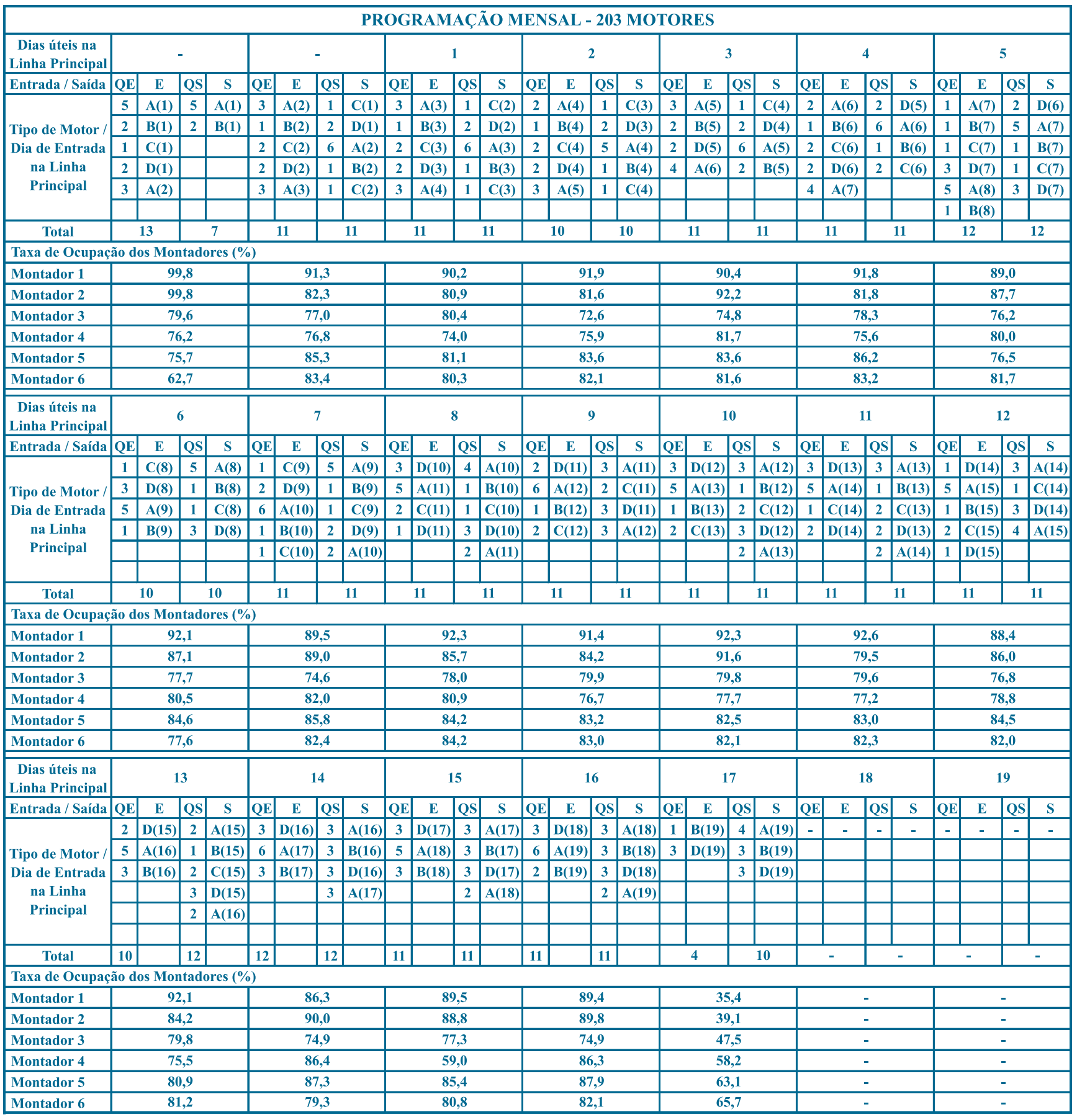


do tipo A, nesta seqüência. Todos estes motores devem estar montados até o primeiro dia útil da programação de montagem na linha principal, com exceção dos últimos três do tipo A, que são demandados na linha principal no segundo dia útil. Nesse mesmo dia, ficam prontos os cinco primeiros motores do tipo $\mathrm{A}$ e os dois do tipo B. A taxa de ocupação dos montadores neste dia foi de: $99,8 \%$ para o montador $1 ; 99,8 \%$ para o montador $2 ; 79,6 \%$ para o montador $3 ; 76,2 \%$ para o montador $4 ; 75,7 \%$ para o montador 5, e 62,7\% para o montador 6 .

Para cada experimento, foi elaborada também uma tabela com os principais resultados globais. A Tabela 2 apresenta os resultados globais obtidos para a linha 1 . Nesta linha foram alocados seis montadores, cada um trabalhando em uma estação, que atendem a demanda do mês analisado correspondente a 203 motores no total. Duas condições para que isso ocorra efetivamente são: a linha 1 deve iniciar sua produção dois dias antes do início das linhas principais e a sequiência de entrada de motores na linha de submontagem deve ser iniciada pelos motores de maiores tempos de montagem seguidos pelos motores de menores tempos de montagem, visando minimizar a perda de tempo na troca do tipo de modelo de motor.

A análise diária da programação mostra que nenhum motor entra na linha de submontagem com mais de três dias de antecedência em relação à sua demanda na linha principal de montagem, atendendo plenamente à condição de antecedência de entrada de motores na linha de submontagem em relação à entrada do motor na linha principal de montagem.

Em relação à formação de estoque na entrada da linha principal, a programação sugerida mostra que $88,2 \%$ dos motores (179) permanecem até um dia no estoque de produtos acabados, atendendo à condição de formação de estoque na entrada da linha principal. Os 11,8\% restantes (24) permanecem dois dias no estoque, o que não causa maiores problemas, pois os motores desta linha são os de menores dimensões entre as três linhas, e o espaço físico destinado para seu estoque suporta este aumento gerado pela permanência destes 24 motores por dois dias.

\section{Linha 2}

Para a linha 2, nos dois experimentos (2 e 3 ) a demanda mensal (36 motores) foi atendida e, a partir da simulação, foram gerados os resultados apresentados nas Tabelas 3 e 4, que correspondem às sugestões para programação diária da linha de submontagem, visando atender diariamente as linhas principais. Nestas sugestões de programação, as colunas "QE" de motores que entram e as colunas "QS" de motores que saem da linha num certo dia foram eliminadas, pois cada linha da programação

Tabela 2: Resultados globais para o experimento 1 da Linha 1.

\begin{tabular}{|c|c|c|}
\hline \multicolumn{3}{|c|}{ Resultados Mix de Motores - Linha 1} \\
\hline Demanda: 203 motores & & Experimento \\
\hline Dias úteis: 19 & & 1 \\
\hline \multirow{6}{*}{ Taxa média de ocupação (\%) } & Montador 1 & 88,2 \\
\hline & Montador 2 & 84,3 \\
\hline & Montador 3 & 75,8 \\
\hline & Montador 4 & 76,8 \\
\hline & Montador 5 & 82,3 \\
\hline & Montador 6 & 79,9 \\
\hline \multicolumn{2}{|c|}{ Início da produção de motores, em relação à demanda da linha principal } & 2 dias antes \\
\hline \multirow{3}{*}{$\begin{array}{l}\text { Quantidade de motores entrando na linha de submontagem, } \\
\text { com relação à demanda da linha principal }\end{array}$} & Até 1 dia antes & $\mathbf{0}$ \\
\hline & Até 2 dias antes & 87 \\
\hline & Até 3 dias antes & 116 \\
\hline \multirow{3}{*}{$\begin{array}{l}\text { Quantidade de motores prontos em estoque } \\
\text { (aguardando entrada na linha principal) }\end{array}$} & 0 dia & 16 \\
\hline & 1 dia & 163 \\
\hline & $2 \operatorname{dias}$ & 24 \\
\hline
\end{tabular}


representa um único motor entrando ou saindo.

Observando-se as Tabelas 3 e 4, percebe-se que, nos dois experimentos, a demanda diária é atendida da mesma forma, não havendo diferenças nas entradas e saídas dos motores na linha.

No experimento 3, porém, considerando-se a condição fornecida pela empresa, na qual dois montadores trabalhando juntos possuem um rendimento de $60 \%$, a taxa de ocupação diária de cada montador é maior do que as taxas de ocupação do experimento 2. Além disso, depen- dendo da integração entre os montadores e do senso de trabalho em equipe, a forma de trabalho do experimento 3 pode aumentar a qualidade do trabalho realizado e diminuir a fadiga dos montadores.

Para esta linha, os experimentos que apresentaram taxas de ocupação abaixo de 50\% indicaram a existência de excesso de montadores. Assim, a determinação da taxa diária de ocupação dos montadores foi importante para a verificação do número mínimo de montadores necessários para o atendimento da demanda mensal.

Tabela 3: Sugestão de programação mensal para a linha 2, para o experimento 2.

\begin{tabular}{|c|c|c|c|c|c|c|c|c|c|c|c|c|c|c|c|c|c|c|c|c|}
\hline \multicolumn{21}{|c|}{ PROGRAMAÇÃO MENSAL - 36 MOTORES } \\
\hline Dias úteis na Linha Principal & \multicolumn{2}{|c|}{-} & \multicolumn{2}{|c|}{1} & \multicolumn{2}{|c|}{2} & \multicolumn{2}{|c|}{3} & \multicolumn{2}{|c|}{4} & \multicolumn{2}{|c|}{5} & \multicolumn{2}{|c|}{6} & \multicolumn{2}{|c|}{7} & \multicolumn{2}{|c|}{8} & \multicolumn{2}{|c|}{9} \\
\hline Entrada / Saída & $\mathbf{E}$ & $\mathbf{S}$ & $\mathbf{E}$ & $\mathbf{S}$ & $\mathbf{E}$ & $\mathbf{S}$ & $\mathbf{E}$ & $\mathbf{S}$ & $\mathbf{E}$ & $\mathbf{S}$ & $\mathbf{E}$ & $\mathbf{s}$ & $\mathbf{E}$ & $\mathbf{S}$ & $\mathbf{E}$ & S & $\mathbf{E}$ & $\mathbf{S}$ & $\mathbf{E}$ & $\mathbf{S}$ \\
\hline \multirow{2}{*}{$\begin{array}{c}\text { Tipo de Motor / Dia de } \\
\text { Entrada na Linha Principal }\end{array}$} & E (1) & E (1) & $F(2)$ & $F(2)$ & F (4) & F (4) & E (6) & E (6) & H (6) & H (6) & F (7) & F (7) & E (8) & E (8) & E (9) & E (9) & H (9) & H (9) & H (10) & H (10) \\
\hline & E (2) & E (2) & G (3) & G (3) & H (5) & H (5) & $F(6)$ & F (6) & E (7) & E (7) & H (7) & H (7) & H (8) & H (8) & F (9) & $F(9)$ & F (10) & F (10) & E (11) & E (11) \\
\hline Total & 2 & 2 & 2 & 2 & 2 & 2 & 2 & 2 & 2 & 2 & 2 & 2 & 2 & 2 & 2 & 2 & 2 & 2 & 2 & 2 \\
\hline \multicolumn{21}{|c|}{ Taxa de Ocupação dos Montadores (\%) } \\
\hline Montador 1 & \multicolumn{2}{|c|}{64,1} & \multicolumn{2}{|c|}{55,9} & \multicolumn{2}{|c|}{66,7} & \multicolumn{2}{|c|}{45,9} & \multicolumn{2}{|c|}{64,2} & \multicolumn{2}{|c|}{67,9} & \multicolumn{2}{|c|}{67,1} & \multicolumn{2}{|c|}{46,8} & \multicolumn{2}{|c|}{46,4} & \multicolumn{2}{|c|}{63,5} \\
\hline Montador 2 & \multicolumn{2}{|c|}{64,6} & \multicolumn{2}{|c|}{46,8} & \multicolumn{2}{|c|}{45,6} & \multicolumn{2}{|c|}{64,2} & \multicolumn{2}{|c|}{68,3} & \multicolumn{2}{|c|}{45,7} & \multicolumn{2}{|c|}{63,2} & 63 & & 67 &, 5 & & 7,0 \\
\hline Dias úteis na Linha Principal & 1 & 0 & 11 & 1 & 12 & 2 & 1. & 3 & 1 & & 15 & & 16 & 6 & 1 & 7 & 1 & 8 & 1 & 19 \\
\hline Entrada / Saída & $\mathbf{E}$ & $\mathbf{s}$ & $\mathbf{E}$ & $\mathbf{s}$ & $\mathbf{E}$ & $\mathbf{S}$ & $\mathbf{E}$ & $\mathbf{S}$ & $\mathbf{E}$ & $\mathbf{S}$ & $\mathbf{E}$ & $\mathbf{S}$ & $\mathbf{E}$ & $\mathbf{S}$ & $\mathbf{E}$ & $\mathbf{S}$ & $\mathbf{E}$ & $\mathbf{S}$ & $\mathbf{E}$ & $\mathbf{S}$ \\
\hline Tipo de Motor / Dia de & F (11) & F (11) & F (12) & F (12) & E (14) & E (14) & H (14) & H (14) & E (15) & E (15) & E (16) & E (16) & E (17) & E (17) & E (18) & E (18) & E (19) & E (19) & - & - \\
\hline Entrada na Linha Principal & H (11) & H (11) & E (13) & E (13) & F (14) & F (14) & H (14) & H (14) & F (15) & F (15) & F (16) & F (16) & & & G (18) & G (18) & & & & \\
\hline Total & 2 & 2 & 2 & 2 & 2 & 2 & 2 & 2 & 2 & 2 & 2 & 2 & 1 & 1 & 2 & 2 & 1 & 1 & - & - \\
\hline Taxa de Ocupação dos Montac & dores $(\%$ & & & & & & & & & & & & & & & & & & & \\
\hline Montador 1 & 67 &, 2 & 63 , & 3,4 & 46 , & 6,6 & 67 & 7,5 & 46 & 6,5 & 47 & & 0, & , 0 & 64 & 4,9 & 63 & 2 & & - \\
\hline Montador 2 & 47 &, 2 & 46, & 6,7 & 63 , &, 5 & 67 & 7,0 & 64 & 4,1 & 64 & & 63 , & 3,2 & 46 & 6,6 & $\mathbf{0}$, & 0 & & - \\
\hline
\end{tabular}

Tabela 4: Sugestão de programação mensal para a linha 2, para o experimento 3.

\begin{tabular}{|c|c|c|c|c|c|c|c|c|c|c|c|c|c|c|c|c|c|c|c|c|}
\hline \multicolumn{21}{|c|}{ PROGRAMAÇÃO MENSAL - 36 MOTORES } \\
\hline Dias úteis na Linha Principal & \multicolumn{2}{|c|}{-} & \multicolumn{2}{|c|}{1} & \multicolumn{2}{|c|}{2} & \multicolumn{2}{|c|}{3} & \multicolumn{2}{|c|}{4} & \multicolumn{2}{|c|}{5} & \multicolumn{2}{|c|}{6} & \multicolumn{2}{|c|}{7} & \multicolumn{2}{|c|}{8} & \multicolumn{2}{|c|}{9} \\
\hline Entrada / Saída & $\mathbf{E}$ & $\mathbf{S}$ & $\mathbf{E}$ & $\mathbf{S}$ & $\mathbf{E}$ & $\mathbf{S}$ & $\mathbf{E}$ & $\mathrm{s}$ & $\mathbf{E}$ & $\mathbf{S}$ & $\mathbf{E}$ & $\mathbf{s}$ & $\mathbf{E}$ & $\mathbf{S}$ & $\mathbf{E}$ & $\mathbf{S}$ & $\mathbf{E}$ & $\mathbf{S}$ & $\mathbf{E}$ & $\mathbf{S}$ \\
\hline \multirow{2}{*}{\begin{tabular}{|c|} 
Tipo de Motor / Dia de \\
Entrada na Linha Principal \\
\end{tabular}} & E (1) & E (1) & F (2) & F (2) & F (4) & F (4) & E (6) & E (6) & H (6) & H (6) & F (7) & F (7) & E (8) & E (8) & E (9) & E (9) & H (9) & H (9) & H (10) & H (10) \\
\hline & E (2) & $E(2)$ & G (3) & G (3) & H (5) & H (5) & $F(6)$ & $F(6)$ & E (7) & E (7) & H (7) & H (7) & H (8) & H (8) & $F(9)$ & F (9) & F (10) & F (10) & E (11) & E (11) \\
\hline Total & 2 & 2 & 2 & 2 & 2 & 2 & 2 & 2 & 2 & 2 & 2 & 2 & 2 & 2 & 2 & 2 & 2 & 2 & 2 & 2 \\
\hline \multicolumn{21}{|c|}{ Taxa de Ocupação dos Montadores (\%) } \\
\hline Montador 1 & \multicolumn{2}{|c|}{78,0} & \multicolumn{2}{|c|}{62,4} & \multicolumn{2}{|c|}{68,5} & \multicolumn{2}{|c|}{66,9} & \multicolumn{2}{|c|}{$\mathbf{8 0 , 0}$} & \multicolumn{2}{|c|}{68,7} & \multicolumn{2}{|c|}{78,7} & \multicolumn{2}{|c|}{67,0} & \multicolumn{2}{|c|}{69,0} & \multicolumn{2}{|c|}{79,1} \\
\hline Montador 2 & \multicolumn{2}{|c|}{77,5} & \multicolumn{2}{|c|}{61,8} & \multicolumn{2}{|c|}{67,9} & \multicolumn{2}{|c|}{66,3} & \multicolumn{2}{|c|}{79,4} & \multicolumn{2}{|c|}{98,0} & \multicolumn{2}{|c|}{78,1} & 66 & 6,4 & 68 & 8,4 & 78 & 8,5 \\
\hline Dias úteis na Linha Principal & & 10 & 1 & 11 & 12 & 12 & & 13 & 14 & 14 & 1 & 5 & 1 & 6 & 1 & 17 & 1 & 8 & 15 & 19 \\
\hline Entrada / Saída & $\mathbf{E}$ & $\mathbf{S}$ & $\mathbf{E}$ & $\mathbf{S}$ & $\mathbf{E}$ & $\mathbf{S}$ & $\mathbf{E}$ & $\mathbf{S}$ & $\mathbf{E}$ & $\mathbf{S}$ & $\mathbf{E}$ & $\mathbf{S}$ & $\mathbf{E}$ & $\mathbf{S}$ & $\mathbf{E}$ & $\mathbf{S}$ & $\mathbf{E}$ & $\mathbf{S}$ & $\mathbf{E}$ & $\mathbf{S}$ \\
\hline Tipo de Motor / Dia de & F (11) & F (11) & $F(12)$ & F (12) & E (14) & E (14) & H (14) & H (14) & E (15) & E (15) & E (16) & E (16) & E (17) & E (17) & E (18) & E (18) & E (19) & E (19) & - & - \\
\hline Entrada na Linha Principal & H (11) & H (11) & E (13) & E (13) & F (14) & F (14) & H (14) & H (14) & F (15) & F (15) & F (16) & F (16) & & & G (18) & G (18) & & & & \\
\hline Total & 2 & 2 & 2 & 2 & 2 & 2 & 2 & 2 & 2 & 2 & 2 & 2 & 1 & 1 & 2 & 2 & 1 & 1 & - & - \\
\hline Taxa de Ocupação dos Mont & adores ( & $(\%)$ & & & & & & & & & & & & & & & & & & \\
\hline Montador 1 & & 9,4 & 67 & 7,2 & 66 & 6,0 & 81 & 1,1 & 67 & 7,3 & 67 & 7,9 & 38 & 8,3 & 67 & 7,4 & 38 & 8,3 & - & - \\
\hline Montador 2 & & 8,8 & $\overline{66}$ & 6,6 & 65 & 5,4 & 80 & 0,6 & 66 & 6,7 & 67 & 7,3 & 38 & 8,0 & $\overline{66}$ & 6,8 & 38 & 8,0 & - & - \\
\hline
\end{tabular}


A Tabela 5 apresenta os resultados globais para os dois experimentos.

Para o atendimento da demanda da linha principal, a linha 2 de submontagem deve iniciar sua produção um dia antes da linha principal. As programações sugeridas pelos dois experimentos atendem plenamente à condição de antecedência de entrada de motores na linha de submontagem em relação à entrada do motor na linha principal de montagem, pois nenhum motor entra na linha de submontagem com mais de três dias de antecedência em relação à sua demanda na linha principal de montagem.

A tabela mostra ainda que, nos dois experimentos, $88,9 \%$ dos motores (32) ficam prontos até um dia antes da sua entrada nas linhas principais, atendendo à condição de estoque na entrada da linha principal.

Os $11,1 \%$ restantes (4) permanecem dois dias no estoque. Assim como para a linha 1, o espaço físico destinado para o estoque de produtos acabados desta linha suporta este aumento gerado pela permanência dos quatro motores por dois dias.

\section{Linha 3}

A Tabela 6 apresenta a sugestão de programação mensal para a linha 3. A previsão de demanda mensal (40 motores) é atendida com a utilização de no mínimo três montadores, conforme a configuração descrita no experimento 4. Assim como nas sugestões de programação para a linha 2, as colunas "QE" de motores que entram e as colunas "QS" de motores que saem da linha num certo dia foram eliminadas, pois cada linha da programação representa um único motor entrando ou saindo.

Para esta linha, assim como para a linha 2, a determinação da taxa diária de ocupação dos montadores foi o índice de orientação para a verificação do número mínimo de montadores necessários para o atendimento da demanda mensal.

\section{s modelos foram gerados de forma que possam ser atualizados rapidamente para a verificação do atendimento de outras previsões de demanda.}

A Tabela 7 apresenta os resultados globais. Para o atendimento da demanda da linha principal, a programação sugerida deve ser iniciada dois dias antes da demanda da linha principal.

Esta tabela mostra que todos os motores entram na linha de submontagem até três dias antes da sua demanda na linha principal, atendendo plenamente à condição de antecedência de entrada na linha de submontagem em relação à demanda na linha principal de montagem.

A condição de formação de estoque na entrada da linha principal também é atendida, pois todos os moto-

Tabela 5: Resultados globais para os experimentos 2 e 3 da linha 2 .

\begin{tabular}{|c|c|c|c|}
\hline \multicolumn{4}{|c|}{ Resultados Mix de Motores - Linha 2} \\
\hline Demanda: 36 motores & & Experimento & Experimento \\
\hline Dias úteis: 19 & & 2 & 3 \\
\hline \multirow{2}{*}{ Taxa média de ocupação (\%) } & Montador 1 & 56,0 & 67,4 \\
\hline & Montador 2 & 56,2 & 68,4 \\
\hline \multicolumn{2}{|c|}{ Início da produção de motores, em relação à demanda da linha principal } & 1 dia antes & 1 dia antes \\
\hline \multirow{3}{*}{$\begin{array}{l}\text { Quantidade de motores entrando na linha de submontagem, } \\
\text { com relação à demanda da linha principal }\end{array}$} & Até 1 dia antes & 17 & 17 \\
\hline & Até 2 dias antes & 15 & 15 \\
\hline & Até 3 dias antes & 4 & 4 \\
\hline \multirow{3}{*}{$\begin{array}{l}\text { Quantidade de motores prontos em estoque } \\
\text { (aguardando entrada na linha principal) }\end{array}$} & 0 dia & 17 & 17 \\
\hline & 1 dia & 15 & 15 \\
\hline & 2 dias & 4 & 4 \\
\hline
\end{tabular}


res permanecem até um dia no estoque de produtos acabados.

\section{CONCLUSÕES}

Uma das capacidades mais exigidas pelo mercado é a agilidade de uma organização em responder às modificações de necessidades deste mercado. Para isso, a organi- zação deve desenvolver estruturas de decisão que permitam o rápido planejamento e replanejamento de seus planos de produção. Neste aspecto, o estudo apresentado mostra-se muito útil para ser incorporado a essas estruturas de decisão.

Os modelos foram gerados de modo a que possam ser atualizados rapidamente para a verificação do atendimento de outras previsões de demanda. Assim, a empresa

Tabela 6: Sugestão de programação mensal para a linha 3.

\begin{tabular}{|c|c|c|c|c|c|c|c|c|c|c|c|c|c|c|c|c|c|c|c|c|c|c|}
\hline \multicolumn{23}{|c|}{ PROGRAMAÇÃO MENSAL - 40 MOTORES } \\
\hline Dias úteis na Linha Principal & \multicolumn{2}{|c|}{-} & \multicolumn{2}{|c|}{-} & \multicolumn{2}{|c|}{1} & \multicolumn{2}{|c|}{2} & \multicolumn{2}{|c|}{3} & \multicolumn{2}{|c|}{4} & \multicolumn{2}{|c|}{5} & \multicolumn{2}{|c|}{6} & \multicolumn{2}{|c|}{7} & \multicolumn{2}{|c|}{8} & \multicolumn{2}{|c|}{9} \\
\hline Entrada / Saída & $\mathbf{E}$ & $\mathbf{S}$ & $\mathbf{E}$ & $\mathbf{s}$ & $\mathbf{E}$ & $\mathbf{S}$ & $\mathbf{E}$ & $\mathbf{S}$ & $\mathbf{E}$ & $\mathbf{S}$ & $\mathbf{E}$ & $\mathbf{S}$ & $\mathbf{E}$ & $\mathbf{S}$ & $\mathbf{E}$ & $\mathbf{s}$ & $\mathbf{E}$ & $\mathbf{S}$ & $\mathbf{E}$ & $\mathbf{S}$ & $\mathbf{E}$ & $\mathbf{S}$ \\
\hline \multirow{3}{*}{$\begin{array}{c}\text { Tipo de Motor (Dia de } \\
\text { Entrada na Linha Principal) }\end{array}$} & I (1) & I (1) & I (2) & I (1) & I (3) & $\mathbf{J}(2)$ & I (4) & $\mathbf{J}(3)$ & I (5) & $\mathbf{J}(4)$ & I (6) & I (5) & I (7) & $\mathbf{J}(6)$ & I ( (8) & I (7) & I (9) & I (8) & I (10) & $\mathrm{J}(9)$ & I (11) & I (10) \\
\hline & I (1) & & $\mathrm{J}(2)$ & I (2) & $\mathbf{J}(3)$ & I (3) & $\mathbf{J}(4)$ & I (4) & I (5) & J (4) & $J(6)$ & $\mathbf{J}(5)$ & I (7) & I (7) & I (8) & I (8) & $\mathbf{J}(9)$ & I (9) & I (10) & I (10) & I (11) & $\mathrm{J}(\mathbf{1 0})$ \\
\hline & & & & & & & $\mathbf{J}(4)$ & & $\mathbf{J}(\mathbf{5})$ & I (5) & & I (6) & & & & & & & $\mathbf{J}(\mathbf{1 0})$ & & & I (11) \\
\hline Total & 2 & 1 & 2 & 2 & 2 & 2 & 3 & 2 & 3 & 3 & 2 & 3 & 2 & 2 & 2 & 2 & 2 & 2 & 3 & 2 & 2 & 3 \\
\hline \multicolumn{23}{|c|}{ Taxa de Ocupação dos Montadores (\%) } \\
\hline Montador 1 & \multicolumn{2}{|c|}{70,6} & \multicolumn{2}{|c|}{67,1} & \multicolumn{2}{|c|}{67,0} & \multicolumn{2}{|c|}{96,8} & \multicolumn{2}{|c|}{99,8} & \multicolumn{2}{|c|}{69,7} & \multicolumn{2}{|c|}{71,6} & \multicolumn{2}{|c|}{67,0} & \multicolumn{2}{|c|}{66,3} & \multicolumn{2}{|c|}{99,8} & \multicolumn{2}{|c|}{75,2} \\
\hline Montador 2 & \multicolumn{2}{|c|}{70,6} & \multicolumn{2}{|c|}{66,9} & \multicolumn{2}{|c|}{66,7} & 96 & & & 9,4 & 69 & 0,5 & 71 & & 69 & 9,8 & 6 & 5,1 & & 9,4 & & 5,0 \\
\hline Montador 3 & 64 & 4,7 & 74 & & 70 & & 72 & 2,4 & & 9,8 & 99 & 9 & 81 & & 76 & 6,9 & 7 & 6,6 & & 7,0 & & 9,9 \\
\hline Dias úteis na Linha Principal & 10 & 10 & 11 & & 1 & 12 & 13 & 3 & & 14 & 1 & 5 & 16 & & 1 & 17 & 1 & 8 & & 19 & & \\
\hline Entrada / Saída & $\mathbf{E}$ & $\mathbf{S}$ & $\mathbf{E}$ & $\mathrm{s}$ & $\mathbf{E}$ & $\mathbf{s}$ & $\mathbf{E}$ & $\mathbf{S}$ & $\mathbf{E}$ & $\mathbf{s}$ & $\mathbf{E}$ & $\mathbf{S}$ & $\mathbf{E}$ & $\mathbf{s}$ & $\mathbf{E}$ & $\mathrm{s}$ & $\mathbf{E}$ & $\mathbf{s}$ & $\mathbf{E}$ & $\mathbf{s}$ & & \\
\hline & I (12) & I (11) & I (13) & I (12) & I (14) & $\mathbf{J}(13)$ & I (15) & I (14) & I (16) & I (15) & I (17) & I (16) & I (18) & I (17) & I (19) & I (19) & - & - & - & - & & \\
\hline $\begin{array}{c}\text { Tipo de Motor (Dia de } \\
\text { Entrada na Linha Principal) }\end{array}$ & I (12) & I (12) & $\mathbf{J}(13)$ & I (13) & I (14) & I (14) & I (15) & I (15) & I (16) & I (16) & I (17) & I (17) & I (19) & I (18) & & I (19) & & & & & & \\
\hline Total & 2 & 2 & 2 & 2 & 2 & 2 & 2 & 2 & 2 & 2 & 2 & 2 & 2 & 2 & 1 & 2 & - & - & - & - & & \\
\hline Taxa de Ocupação dos Monta & idores $\left({ }^{\circ}\right.$ & & & & & & & & & & & & & & & & & & & & & \\
\hline Montador 1 & 71 & 1,0 & 66 & & 72 & 2,6 & 70 & 0,8 & & 0,9 & 72 & 2,3 & 70 & & 36 & 6,3 & & - & & - & & \\
\hline Montador 2 & 70 & 0,8 & 66 & & 72 & 2,4 & 70 & 9,6 & & 0,7 & 72 & 2,0 & 70 & & 36 & 6,2 & & - & & - & & \\
\hline Montador 3 & 83 & 3,1 & 75 & & 70 & 0,1 & 74 & 4,3 & 75 & 5,9 & 74 & $\overline{1,6}$ & 77 & & 51 & 1,2 & & - & & - & & \\
\hline
\end{tabular}

Tabela 7: Resultados globais para o experimento 4 da linha3.

\section{Resultados Mix de Motores - Linha 3}

Demanda: 40 motores

Dias úteis: 19

Taxa média de ocupação (\%)

Experimento

\begin{tabular}{|c|c|c|}
\hline Dias úteis: 19 & & 4 \\
\hline \multirow{3}{*}{ Taxa média de ocupação (\%) } & Montador 1 & 72,7 \\
\hline & Montador 2 & 72,6 \\
\hline & Montador 3 & 77,1 \\
\hline \multicolumn{2}{|c|}{ Início da produção de motores, em relação à demanda da linha principal } & 2 dias antes \\
\hline \multirow{3}{*}{$\begin{array}{l}\text { Quantidade de motores entrando na linha de submontagem, } \\
\text { com relação à demanda da linha principal }\end{array}$} & Até 1 dia antes & $\mathbf{0}$ \\
\hline & Até 2 dias antes & 39 \\
\hline & Até 3 dias antes & 1 \\
\hline \multirow{3}{*}{$\begin{array}{l}\text { Quantidade de motores prontos em estoque } \\
\text { (aguardando entrada na linha principal) }\end{array}$} & 0 dia & 20 \\
\hline & 1 dia & 20 \\
\hline & 2 dias & $\mathbf{0}$ \\
\hline
\end{tabular}


pode planejar antecipadamente e com uma maior margem de segurança a programação e alocação da mão-deobra em um horizonte de médio prazo, que englobe alguns meses de previsão.

A determinação da taxa diária de ocupação de cada montador pode ser útil no apoio ao planejamento de outras atividades para estes funcionários ao longo do mês, tais como limpeza do local de trabalho, reuniões, treinamentos e cursos. O estudo desenvolvido pode auxiliar na racionalização do número de montadores, especialmente quando a equipe de montadores possuir capacidades multifuncionais, o que facilita a alteração de alocação dos montadores nos postos de trabalho ao longo dos meses.

\section{Artigo recebido em 20/12/2002 Aprovado para publicação em 15/07/2003}

\section{- Bibliografia}

AL-AOMAR, R. Product-mix analysis with discrete event simulation. In: Winter Simulation Conference, Proceedings, $\mathrm{p}$. 1385-1392, 2000.

ARTTO, K. A.; LEHTONEN, J. M.; SARANEN, J. Managing projects frontend: incorporating a strategic early view to project management with simulation. International Journal of Project Management, v. 19, p. 255-264, 2001.

ASKIN, R. G.; STANDRIDGE, C. R Modeling and analysis of manufacturing systems. New York: Wiley, 1993. 461 p.

BENGU, G. A simulation-based scheduler for flexible flowlines. International Journal of Production Research, v. 32, n. 2, p. 321-344, 1994.
COSTA, M. T.; FERREIRA, J. S. A simulation analysis of sequencing rules in a flexible flowline. European Journal of Operational Research, v. 119, p. 440 450, 1999.

FOKKERT, J. I. Z.; KOK, T. G. The mixed and multi model line balancing problem: a comparison. European Journal of Operational Research, v. 100, p. 399-412, 1997.

FUH, J. Y.; WONG, Y. S.; YEE, C. Y.; ZHUANG, L.; NEO, K. S. Modeling, analysis and simulation for the design of a robotic assembly system. Computer Integrated Manufacturing Systems, v. 9, n. 1, p. 19-31, 1996
GHOSH, S.; GAGNON, R. J. A comprehensive literature review and analysis of the design, balancing and scheduling of assembly systems. International Journal of Production Research, v. 27, n. 4, p. 637-670, 1989.

HE, D. W.; KUSIAK, A. Designing an assembly line for modular products. Computers \& Industrial Engineering, v. 34, n. 1, p. 37-52, 1998.

HO, Y.; LEE, C. C.; MOODIE, C. L. Two sequence-pattern, matching-based, flow analysis methods for multi-flowlines layout design. International Journal of Production Research, v. 31, n. 7, p. 1557 1578, 1993.
KHAN, A.; DAY, A. J. A knowledge based design methodology for manufacturing assembly lines. Computers \& Industrial Engineering, v. 41, p. 441-467, 2002.

SIANESI, A. An analysis of the impact of plant and management variables in a multi-stage, mixed-model production systems. International Journal of Production Economics, 56-57, p. 575-585, 1998.

VAIDYANATHAN, B. S.; PARK, Y. H.; MILLER, D. M. Application of discrete event simulation in production scheduling. In: Winter Simulation Conference, Proceedings, p. 965-971, 1998. 